\title{
Modified spectrum autointerferometric correlation (MOSAIC) for single-shot pulse characterization
}

\author{
Daniel A. Bender* and Mansoor Sheik-Bahae \\ Department of Physics and Astronomy, Optical Science and Engineering Program, University of New Mexico, \\ Albuquerque, New Mexico 87131, USA \\ *Corresponding author: dnbender@unm.edu
}

Received July 10, 2007; accepted August 10, 2007; posted August 22, 2007 (Doc. ID 85149); published September 25, 2007

A method for generation of the modified spectrum autointerferometric correlation that allows single-shot pulse characterization is demonstrated. A sensitive graphical representation of the ultrashort pulse phase quality is introduced that delineates the difference between the presence of temporal and spectral phase distortions. Using these schemes, full-field reconstruction of ultrashort laser pulses is obtained in real time using an efficient iterative technique. (c) 2007 Optical Society of America

OCIS codes: $320.7100,140.7090$.

Detailed characterization of ultrashort laser pulses is vital for many applications. There exist many pulse characterization techniques with varying experimental and/or computational complexity. These range from interferometric autocorrelation (IAC) [1] to fullfield reconstruction techniques such as frequencyresolved optical gating (FROG) [2] and spectral shear interferometry for direct electric field reconstruction (SPIDER) [3]. Adaptive pulse characterization techniques such as multiphoton intrapulse interference phase scan (MIIPS) [4] have been successful not only in retrieving the electric field but in compensating the spectral phase to achieve transform-limited pulses. In many applications, full-field reconstruction may not be required, although sensitive real-time information on phase distortions due to the presence of chirp and dispersion is still essential. Modified spectrum autointerferometric correlation (MOSAIC) was developed to address this need using the information contained in an IAC trace and has been shown to be far more sensitive to pulse chirp than a traditional IAC [5]. The new advances in the implementation of MOSAIC are the following.

(a) Single-shot characterization using a combination of fringe-free (noninterferometric) autocorrelation and second-harmonic spectrum

(b) A hybrid graphical representation that distinguishes between spectral and temporal phase distortions

(c) Real-time full-field reconstruction using the above schemes with an efficient sequential search algorithm

Naganuma et al. showed that the pulse spectrum and IAC provide a sufficient dataset to uniquely reconstruct the complex electric field, with only a timedirection ambiguity [6]. Retrieval techniques such as phase and intensity from correlation and spectrum only (PICASO) [7] make use of a similar three envelope dataset. The usefulness of MOSAIC was extended by use of homodyne operation and signal averaging [8]. High-fidelity MOSAIC traces were recovered in the presence of extreme noise. This fringe-free averaging technique measures the upper and lower MOSAIC envelopes with a very high signal-to-noise ratio (SNR) and allows us to clearly distinguish pulses that produce essentially identical IAC traces [9]. The fringe-free MOSAIC technique has recently been used to characterize ultrashort pulses in the mid-IR [10].

The increased SNR found on averaged MOSAIC traces extends the utility of all retrieval techniques using the dataset outlined by Naganuma et al. [6]. By combining MOSAIC data with the first-order interferogram and performing additional analysis, the spectral phase of the electric field can be recovered [11]. A number of synthesized pulses of varying complexity were shown to be reconstructed successfully using an iterative method. Retrieval methods using MOSAIC are similar to PICASO but provide the advantages of time-domain signal averaging and straightforward visual chirp interpretation.

The principle of computing a MOSAIC can be described in the frequency domain as follows: a secondorder IAC waveform with a fringe frequency $\Omega$ is Fourier transformed to generate a spectrum. Spectral filtering is then performed to remove the $\Omega$ component and amplify the $2 \Omega$ component by a factor of 2. An inverse Fourier transform generates a new time-domain signal known as a fringe-resolved MOSAIC [5]. The upper envelope is replaced by the intensity profile while the lower envelope-identified by two shoulders above the baseline-provides background-free phase information. Examples are shown in Fig. 1 (solid curves). The presence of shoulders is a signature of temporal pulse chirp while a flat baseline is an indication of a flat temporal phase [12].

In the (delay) time-domain analysis, the maximum and minimum envelopes of MOSAIC are given by the intensity autocorrelation, $g(\tau)=\int f(t) f(t+\tau) \mathrm{d} t$, and the difference computation, $S_{\min }=g(\tau)-\left|g_{p}(\tau)\right|$, respectively [8]. The amplified $2 \Omega$ component of the IAC is expressed as

$$
g_{p}(\tau)=\int f(t) f(t+\tau) e^{2 i[\phi(t)-\phi(t+\tau)]} \mathrm{d} t,
$$


which is also the envelope of the second-harmonic field autocorrelation. The temporal chirp is represented by $\phi(t)$, while $f(t)$ is the intensity of the pulse. The second-harmonic field autocorrelation is related to the second-harmonic spectrum by

$$
\left|g_{p}(\tau)\right|=\left|F^{-1}\left(\left|E^{2}(\Omega)\right|^{2}\right)\right|,
$$

where the second-harmonic power spectrum is represented by $\left|E^{2}(\Omega)\right|^{2}$ and $F^{-1}$ denotes the inverse Fourier transform operation. For single-shot arrangements, fringe-resolved second-order IAC traces are not practical to produce. The method we present here overcomes this obstacle by making use of the intensity autocorrelation and second-harmonic generation (SHG) spectrum. We call this envelope-MOSAIC or E-MOSAIC, which can be very useful for characterizing ultrashort pulses from low repetition rate (approximately kilohertz) amplified laser systems.

In a proof-of-principle demonstration, E-MOSAIC is compared with MOSAIC in the following experiment. A second-order IAC is produced with modelocked $85 \mathrm{fs}$ Ti:sapphire laser pulses centered at a wavelength of $825 \mathrm{~nm}$ using SHG. An SHG spectrum is collected by blocking the delay arm and routing the frequency-doubled output to an Ocean Optics HR4000 spectrometer with an $8 \mu \mathrm{m}$ optical fiber. The inverse Fourier transform of the second-harmonic power spectrum is computed over a spectral bandwidth consistent with the total time delay of the intensity autocorrelation. The resulting time-domain trace is normalized to the intensity autocorrelation and subtracted from the intensity autocorrelation to produce $S_{\min }$ [see Fig. 1(a), open circles]. This result is compared with $S_{\min }$ and the intensity autocorrelation obtained from the second-order IAC using the same experimental setup [Fig. 1(a), solid lines]. The excellent agreement between the two approaches validates the fidelity of E-MOSAIC. Next, we deliberately chirp the laser pulse prior to the autocorrelator by passing it through a $2 \mathrm{~mm}$ thick ZnSe window and perform the same operations described above. Results presented in Fig. 1(b) again show clear agreement.

Temporal chirp interpretation from a MOSAIC trace is typically straightforward, as evidenced by the appearance of shoulders in the minimum envelope. As Fourier analysis indicates, however, spectral
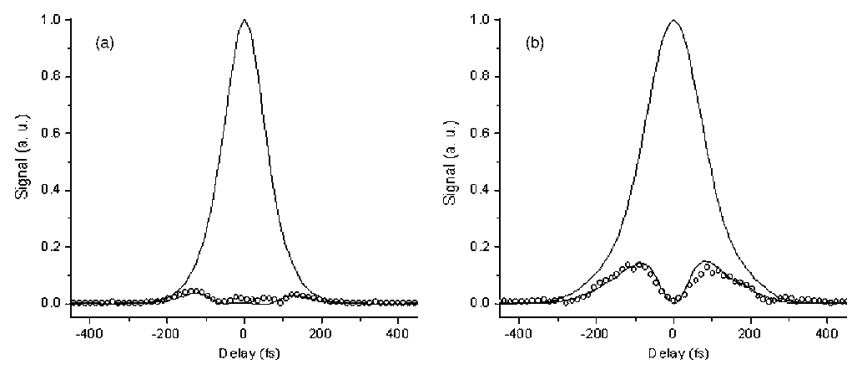

Fig. 1. MOSAIC traces obtained from (a) 85 fs Ti:sapphire laser and (b) intentionally chirping the laser pulse with $2 \mathrm{~mm}$ of ZnSe. MOSAIC rendered from an SHG spectral measurement (circles) and a second-order IAC (solid curve). phase distortion (dispersion) does not necessarily imply temporal phase distortion (chirp). For example, a pulse having a symmetric spectrum and only thirdorder dispersion (TOD) will exhibit a flat MOSAIC minimum but is not transform limited. Conversely, in the instance of an asymmetric spectrum and flat spectral phase, the MOSAIC trace will exhibit a nonzero minimum envelope while the intensity autocorrelation is bandwidth limited. To accommodate these situations, we introduce an enhanced representation of the MOSAIC trace that is distinctly sensitive to both spectral dispersion and temporal chirp in pulse distortions. The added sensitivity requires the pulse spectrum in addition to second-order IAC. In rapidscan IAC schemes, this can be obtained by adding a linear detector to the same interferometer $[8,11]$. This enhanced representation is displayed in the form of a hybrid (H)-MOSAIC. An H-MOSAIC trace is produced by assigning a flat phase across the measured pulse spectrum and computing the transformlimited intensity autocorrelation, $g^{T L}(\tau)$, which is then normalized to $g(\tau)$ at $\tau=0$. The difference between the transform-limited and measured intensity autocorrelation is computed. A symmetric doublehump appears for broadened pulses while a transform-limited pulse is flat. Accounting for symmetry with respect to zero delay, we take the average of both halves in both the difference computation and the measured lower MOSAIC envelope, $g(\tau)-\left|g_{p}(\tau)\right|$. We define the lower envelope of H-MOSAIC as

$$
S_{H Y B}(\tau)=\left\{\begin{array}{ll}
g(\tau)-g^{T L}(\tau) & \text { for } \tau<0 \\
g(\tau)-\left|g_{p}(\tau)\right| & \text { for } \tau \geq 0
\end{array} .\right.
$$

The upper envelope of H-MOSAIC is $g(\tau)$. The lower envelope of H-MOSAIC is sensitive to spectral dispersion for $\tau<0$ and temporal chirp for $\tau \geq 0$. It is important to note the nonorthogonality of the H-MOSAIC peaks, i.e., in general dispersion can give rise to a temporal chirp and vice versa. In Fig. 2 we

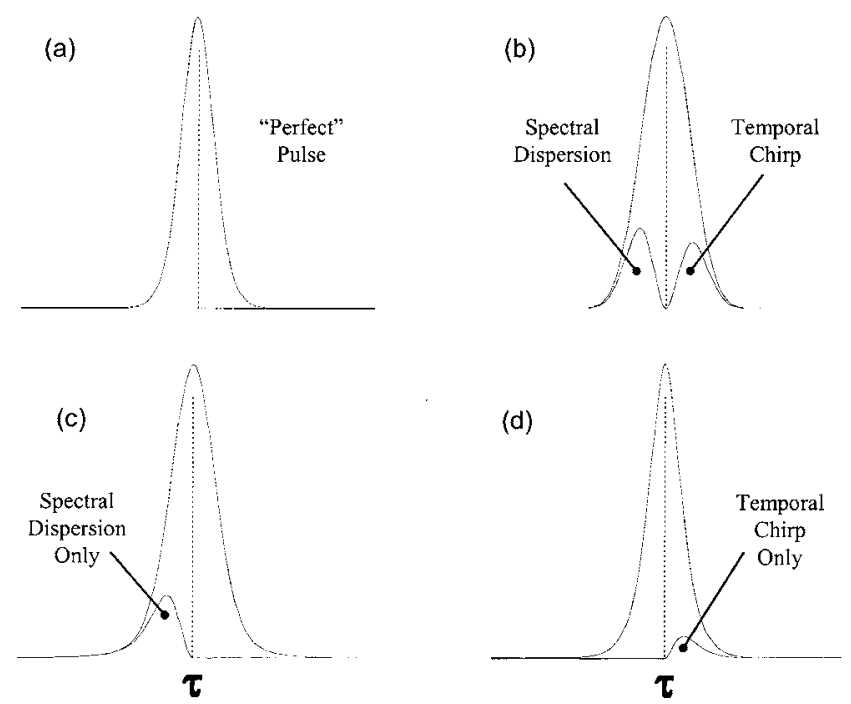

Fig. 2. Simulation of H-MOSAIC for a pulse having a symmetric spectrum with (a) no dispersion, (b) GVD, (c) TOD, and (d) asymmetric spectrum with no dispersion. The dashed line indicates zero delay. 
present H-MOSAIC simulations for the special cases of a pulse having a symmetric spectrum with (a) no dispersion, (b) group-velocity dispersion (GVD) only, and (c) TOD only. The pulse distinguished by the trace in (a) can be regarded as the "perfect pulse"; symmetric in time and frequency, and without chirp or dispersion. Any small deviation from this symmetry will appear as shoulders in the H-MOSAIC traces. Note that in case (c) when only TOD is present we will have $g(\tau)-\left|g_{p}(\tau)\right|=0$. Displayed in part (d) of Fig. 2 is an H-MOSAIC produced from an asymmetric spectrum and flat spectral phase. This is transform limited, which leads to $S_{H Y B}(\tau)=0$ for $\tau$ $<0$ because of the flat spectral phase. We note that there is no new information in H-MOSAIC that is not already present in a MOSAIC trace (or IAC) and spectrum. However, an H-MOSAIC trace extracts and renders such information in an easily identifiable graphical representation.

The H-MOSAIC trace is a visual representation of the dataset used in one-dimensional retrieval schemes. Experimental reconstruction using MOSAIC and the pulse spectrum has been demonstrated using an iterative line minimization technique [11]. In this reconstruction method, all points in the spectral phase are optimized individually at the expense of processing time. We can reduce the processing time $\sim 7 \times$ by analyzing phase with a fourth-order Taylor-series expansion and adjusting the coefficients. Our retrieval algorithm is implemented by sequential optimization of the Taylorseries coefficients and works by first optimizing GVD, then TOD and if needed fourth-order dispersion. This sequence accounts for the prominence of the lowerorder spectral phase coefficients encountered in realistic pulses and gives direct insight to the order of dispersion on a pulse possessing a Taylor expandable phase.

We use the sequential optimization technique to reconstruct the electric field from the measured pulse spectrum and MOSAIC of Fig. 1(b). An iterative simplex algorithm minimizes the root-mean-square (rms) error, $\Delta$. Minimization of the rms defines convergence of the algorithm. The measured spectrum and retrieved spectral phase from retrieval (R)-MOSAIC are shown in Fig. 3(a). Execution of the sequential reconstruction algorithm using 128 points is accomplished on a laptop computer in less than $1 \mathrm{~s}$ with a $1.8 \mathrm{GHz}$ Centrino Duo processor for $\Delta$ $=0.0072$. This minimum achievable rms error is set by experimental noise and higher-order phase terms not accounted for in the Taylor-series phase expansion.

Further reduction of rms error can be obtained by individual optimization of each point in the spectral phase using a line-search method similar to [11]. Our line-search algorithm is seeded with the sequential reconstruction result and run until a new minimum achievable rms is found. The retrieved phase and re-
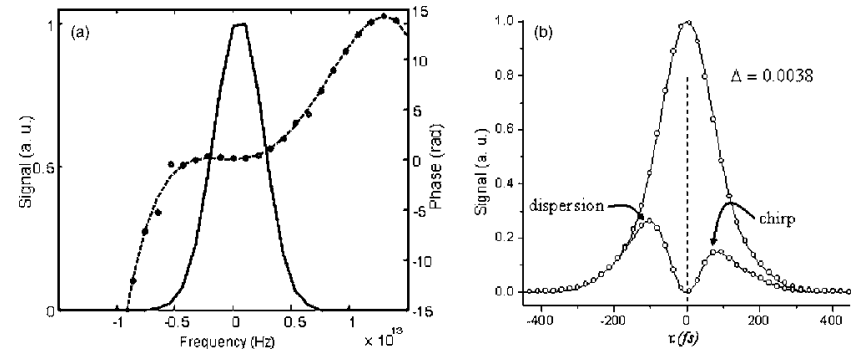

Fig. 3. (a) Measured spectrum (solid curve) and retrieved phase (dashed curve) from the R-MOSAIC algorithm. The retrieved phase from an individual point line search is reproduced (dots). (b) Experimental H-MOSAIC (solid curve) and reconstructed H-MOSAIC (circles) from the phase (dots) and measured spectrum of (a).

constructed H-MOSAIC trace are depicted in Fig. 3(a) (dots) and Fig. 3(b) (circles), respectively. Processing time increases to $\sim 30 \mathrm{~s}$, giving a reduction of rms error from 0.0072 to 0.0038 . We find this accuracy improvement to be of little practical benefit.

In summary, we have shown that the MOSAIC pulse characterization algorithm can be implemented in a single-shot scheme (provided the SNR is sufficiently high) that we call E-MOSAIC. We determine the location of phase distortions using a selective H-MOSAIC trace. Real-time phase retrieval possessing only time-direction ambiguity was experimentally demonstrated for pulses having spectral dispersion. MOSAIC software is available for free download at http://www.optics.unm.edu/sbahae/.

The authors wish to acknowledge helpful discussions with B. Yellampalle and M. Hasselbeck. Support provided through National Science Foundation awards ECS-0100636 and DGE-0114319 (Integrative Graduate) is gratefully acknowledged.

\section{References}

1. J.-C. Diels, J. J. Fontaine, I. C. McMichael, and F. Simoni, Appl. Opt. 24, 1270 (1985).

2. D. J. Kane and R. Trebino, IEEE J. Quantum Electron. 29, 571 (1993).

3. C. Iaconis and I. A. Walmsley, IEEE J. Quantum Electron. 35, 501 (1999).

4. V. V. Lozovoy, I. Pastirk, and M. Dantus, Opt. Lett. 29, 775 (2004).

5. T. Hirayama and M. Sheik-Bahae, Opt. Lett. 27, 860 (2002).

6. K. Naganuma, K. Modi, and H. Yamada, IEEE J. Quantum Electron. 25, 1225 (1989).

7. J. W. Nicholson and W. R. Rudolph, J. Opt. Soc. Am. B 19, 330 (2002).

8. D. A. Bender, M. P. Hasselbeck, and M. Sheik-Bahae, Opt. Lett. 31, 122 (2006).

9. J. Chung and A. M. Weiner, IEEE J. Sel. Top. Quantum Electron. 7, 656 (2001).

10. S.-H. Shim, D. B. Strasfeld, and M. T. Zanni, Opt. Express 14, 13120 (2006).

11. B. Yellampalle, R. D. Averitt, and A. J. Taylor, Opt. Express 14, 8890 (2006).

12. M. Sheik-Bahae, Opt. Lett. 22, 399 (1997). 